Circ Arrhythm Electrophysiol. 2015 April ; 8(2): 409-419. doi:10.1161/CIRCEP.114.002065.

\title{
Arrhythmogenic Remodeling of $\beta_{2}$ versus $\beta_{1}$ Adrenergic Signaling in the Human Failing Heart
}

\author{
Di Lang, PhD ${ }^{1}$, Katherine Holzem, MSc ${ }^{1}$, Chaoyi Kang, BS ${ }^{1}$, Mengqian Xiao, BS ${ }^{1}$, Hye Jin \\ Hwang, MD,PhD ${ }^{1}$, Gregory A. Ewald, MD $^{2}$, Kathryn A. Yamada, PhD $^{2}$, and Igor R. Efimov, \\ $\mathrm{PhD}^{1,2,3,4}$ \\ ${ }^{1}$ Department of Biomedical Engineering, Washington University in St. Louis \\ ${ }^{2}$ Department of Medicine, Washington University School of Medicine, St. Louis, MO \\ ${ }^{3}$ L'Institut de Rythmologie et Modélisation Cardiaque LIRYC, Université de Bordeaux, Bordeaux, \\ France; \\ ${ }^{4}$ Moscow Institute of Physics and Technology, Moscow, Russia
}

\begin{abstract}
Background-Arrhythmia is the major cause of death in patients with heart failure, for which $\beta$ adrenergic receptor (AR) blockers are a mainstay therapy. But the role of $\beta$-adrenergic signaling in electrophysiology and arrhythmias has never been studied in human ventricles.

Methods and Results-We used optical imaging of action potentials (AP) and $\left[\mathrm{Ca}^{2+}\right]_{\mathrm{i}}$ transients $(\mathrm{CaT})$ to compare the $\beta_{1}$ - and $\beta_{2}$-adrenergic responses in left ventricular wedge preparations of human donor and failing hearts. $\beta_{1}$-stimulation significantly increased conduction velocity $(\mathrm{CV})$, shortened AP duration (APD), CaT duration $(\mathrm{CaD})$ in donor but not failing hearts, due to desensitization of $\beta_{1}$-AR in heart failure. In contrast, $\beta_{2}$-stimulation increased $\mathrm{CV}$ in both donor and failing hearts but shortened APD only in failing hearts. $\beta_{2}$-stimulation also affected transmural heterogeneity in APD but not in $\mathrm{CaD}$. Both $\beta_{1}$ - and $\beta_{2}$-stimulation augmented the vulnerability and frequency of ectopic activity and enhanced substrates for ventricular tachycardia in failing, but not donor, hearts. Both $\beta_{1}$ - and $\beta_{2}$-stimulation enhanced Purkinje fiber automaticity, while only $\beta_{2}$-stimulation promoted Ca-mediated premature ventricular contractions in heart failure.
\end{abstract}

Conclusions-During end-stage heart failure, $\beta_{2}$-stimulation creates arrhythmogenic substrates via $\mathrm{CV}$ regulation and transmurally heterogeneous repolarization. $\beta_{2}$-stimulation is, therefore, more arrhythmogenic than $\beta_{1}$-stimulation. In particular, $\beta_{2}$-stimulation increases the transmural difference between $\mathrm{CaD}$ and $\mathrm{APD}$, which facilitates the formation of delayed afterdepolarizations.

\section{Keywords}

arrhythmia (mechanisms); heart failure; calcium; adrenergic receptor; delayed afterdepolarization; $\beta_{1} ; \beta_{2}$ adrenergic receptor

Correspondence: Igor R. Efimov, PhD, Washington University, 390E Whitaker Hall, One Brookings Drive, St. Louis, MO 63130, Tel: 314-935-8612, Fax: 314-935-7448, igor@wustl.edu. 


\section{Introduction}

Increased $\beta$-adrenergic sympathetic activity is a hallmark of heart failure and often leads to ventricular remodeling and impaired cardiac contractile function. Up to $50 \%$ of heart failure mortality is sudden and due to arrhythmias. $\beta_{1}$ or non-selective adrenergic blockers can reverse ventricular remodeling and reduce mortality in heart failure patients. ${ }^{1}$ Although the antiarrhythmic effect of $\beta$-blockers has long been recognized, ${ }^{2}$ the underlying mechanism is incompletely understood. ${ }^{3}$ So far, the effect of $\beta$-adrenergic signaling on electrophysiology and arrhythmias has never been studied in tissue preparations from failing and non-failing human hearts.

$\beta$-adrenergic stimulation can lead to sarcoplasmic reticulum (SR) Ca overload, spontaneous SR Ca release and delayed afterdepolarization (DAD). ${ }^{4}$ In addition, $\beta$-receptors regulate multiple ion channels via the cyclic adenosine monophosphate/protein kinase A (cAMP/ PKA) cascade, which is electrogenic and potentially arrhythmogenic. However, cellular studies cannot fully explain arrhythmogenesis and arrhythmia maintenance at the tissue level. Potential arrhythmogenic substrates due to $\beta$-stimulation include abnormal impulse conduction, altered repolarization dynamics, and transmural heterogeneity in repolarization. The human ventricular free wall is comprised of three layers: subendocardium, midmyocardium, and subepicardium. These layers possess different intrinsic electrical properties that account for activation delays and synchronized ventricular repolarization. ${ }^{5}$ In heart failure, these properties are altered due to molecular and structural remodeling, including remodeling of $\beta$-adrenergic receptors (AR), and may set the stage for triggered arrhythmias, as seen in patients with heart failure.

Two dominant subtypes of $\beta$-AR are $\beta_{1}$ - and $\beta_{2}$-AR. While $\beta_{1}$-AR predominates in the human myocardium, $\beta_{2}$-AR is equally important due to its more efficient adenylyl cyclase coupling and endogenous role in cardiac regulation. ${ }^{6}$ Recently, the use of non-selective $\beta$ blockers or $\beta_{2}$-blockers was advocated by clinical trials and studies ${ }^{7,8}$, emphasizing the importance of $\beta_{2}$-adrenergic signaling in heart failure. Although the effects of remodeling the $\beta_{1} / \beta_{2}$-adrenergic system on contractility have been studied in human trabeculae, ${ }^{9}$ the effect $\beta_{1} / \beta_{2}$-remodeling on electrophysiology and arrhythmogenesis in the left ventricular wall of the human failing heart is unknown.

We hypothesized that in end-stage heart failure, $\beta_{1}$ - and $\beta_{2}$-adrenergic signaling are differentially remodeled and have different effects on arrhythmogenesis. To compare $\beta_{1}$ and $\beta_{2}$ selective adrenergic effects, we assessed EC coupling using dual optical mapping of AP and $\mathrm{CaT}$ in left ventricular wedge preparations from human failing $(\mathrm{F})$ and donor $(\mathrm{D})$ hearts. Our data indicate that $\beta_{2}$-stimulation, in contrast to $\beta_{1}$-stimulation, induces transmural heterogeneity in repolarization and sets the stage for arrhythmias. Furthermore, we show that $\beta_{2}$-AR signaling enables the formation of DADs by increasing the difference between $\mathrm{CaD}$ and $\mathrm{APD}$, suggesting an underlying mechanism of triggered arrhythmias, such as those observed in patients with heart failure. 


\section{Material and Methods}

\section{Experimental Protocol}

Procurement and use of human myocardium was approved by the Institutional Review Board of Washington University School of Medicine. Non-ischemic end-stage failing human hearts $(\mathrm{F}, \mathrm{n}=8)$ were procured at the time of transplantation at Barnes-Jewish Hospital. Nonfailing donor hearts $(\mathrm{D}, \mathrm{n}=8)$ were procured at Mid-America Transplant Services (St. Louis, MO) and were used as controls. The clinical data of patients are shown in Supplemental Table 1.

The arterially perfused LV wedge preparations (Supplemental Figure 1) and dual optical mapping were described previously ${ }^{10,11}$ and in Supplemental Methods. Fluorescent probes RH237 and Rhod-2AM were utilized for AP and CaT, respectively. We used selective $\beta_{2^{-}}$ AR agonist Procaterol $(1 \mu \mathrm{mol} / \mathrm{L})^{12}$, selective $\beta_{1}$-AR agonist Xamoterol $(1 \mu \mathrm{mol} / \mathrm{L})^{13}$, and non-specific $\beta$-AR agonist Isoproterenol (ISO, 100nmol/L) (Tocris Bioscience, Ellisville, MO) (Supplemental Figure 2). The agonists were applied at saturating concentrations for maximum effective activation of the receptors ${ }^{12,13}$. Complete washout evaluated by the recovery of $\mathrm{CV}$, APD and $\mathrm{CaD}$ was applied between Xamoterol and Procaterol.

\section{Statistical Analysis}

For studies in which baseline and $\beta$-stimulation measures were compared, we conducted analyses with a mixed random effects repeated measures model with group, time, and groupby-time interaction as fixed effects. For each analysis, several covariance structures were fit, and the fit with the smallest Bayesian Information Criteria score was used, which was the Toeplitz matrix structure. For experiments in which transmural layers were analyzed separately, we used a mixed random effects, split-plot study design to account for layers having different degrees of freedom relative to the group. Post-hoc t-tests were then carried out with Bonferroni correction. We considered $\mathrm{p}<0.05 /$ (number of pairwise tests) to be statistically significant.

In Supplemental Methods, we provide detailed data analysis and describe experimental protocols and methods, including Western blot, q-PCR, and immunofluorescence.

\section{Results}

\section{Effects of $\beta_{2}$ - versus $\beta_{1}$-AR stimulation on arrhythmia substrates in heart failure}

Effects of $\beta_{2}$-AR stimulation on $\mathbf{C V}-$ We quantified anisotropy as the ratio between longitudinal conduction velocity $\left(\mathrm{CV}_{\mathrm{L}}\right)$ and transverse conduction velocity $\left(\mathrm{CV}_{\mathrm{T}}\right)^{14}$ (Figure 1). In both donor and failing hearts, $C V_{T}$ did not demonstrate a physiologically significant difference after $\beta_{1}$ - or $\beta_{2}$-stimulation, though there was a statistically significant difference for $\beta_{2}$-stimulation in failure (Figure 1C-D). Both $\beta_{1}$ and $\beta_{2}$ agonists dramatically increased $\mathrm{CV}_{\mathrm{L}}$ in donor hearts. In failing hearts, only $\beta_{2}$, but not $\beta_{1}$, agonist increased $\mathrm{CV}_{\mathrm{L}}$ (Figure $1 \mathrm{E}-\mathrm{F})$. The quantitative analysis of velocity changes are shown in Supplementary Figure 3. Accordingly, anisotropy decreased with $\beta_{1}$-stimulation and increased with $\beta_{2}$-stimulation in 
failing hearts; however, in donor hearts conduction anisotropy is only increased significantly during $\beta_{2}$-stimulation (Figure 1G-H).

\section{$\beta_{2}$-stimulation increasedtransmural heterogeneity of repolarization in failing} hearts-To assess the effect of $\beta$-stimulation on repolarization we quantified APD at $80 \%$ of repolarization in the subendocardium (endo), midmyocardium (mid), and subepicardium (epi) (Figure 2). Classification of all layers is shown in Supplemental Figure 2. As previous studies have also shown ${ }^{15}$, we determined that APD in failing hearts, measured at different pacing rates in the absence or presence of $\beta$-stimulation, is significantly longer than APD in donor hearts (Figure 2B). Both $\beta$-agonists shortened APD in both groups. However, for the different layers, the agonists reduced APD to varying degrees (Figure 2C and Supplemental Table 2). This layer-dependent APD reduction increased the APD difference between endocardium and epicardium $\left(\mathrm{APD}_{80 \text { endo }}-\mathrm{APD}_{80 \mathrm{epi}}\right)$ dramatically more in failing hearts than in donor hearts (Figure 2D).

We quantified the layer-dependent APD reduction $(\triangle \mathrm{APD})$ at $1 \mathrm{~Hz}$ pacing in response to both $\beta$-agonists as the difference of APD values between baseline and in the presence of $\beta$ agonists (Figure $3 \mathrm{~A}$ ). $\triangle$ APD maps revealed that $\beta_{1^{-}}$and $\beta_{2}$-stimulation each resulted in different spatial patterns of APD reduction (Figure 3B).

In donor hearts, $\beta_{2}$-agonist stimulation caused decreasing $\triangle$ APDs (increasing reductions in APD) from the epicardium toward the endocardium. The opposite is seen in failing hearts, where $\beta_{2}$-agonist treatment caused increasing $\triangle$ APDs (decreasing reductions in APD) from the endocardium to the epicardium (Figure $3 \mathrm{C}$ ). Taken together, the $\triangle \mathrm{APD}$ effect sizes between donor and failing hearts were greatest at the epicardium and decreased toward the endocardium. Strongly significant group-layer interactions for $\triangle$ APD values $(\mathrm{p}<0.0001)$ highlight the differences in effect sizes between donor and failing hearts (Figure 3C). In failing hearts, the average APD reduction caused by $\beta_{2}$-agonist in the transmural wall is greater compared with that in donor hearts. This suggests that the $\beta_{2}$-AR pathway is sensitized in heart failure. $\beta_{1}$-agonist treatment produced APD reductions that were most pronounced in the endocardium and decreased toward the epicardium of donor hearts. In failing hearts, a modest and uniform APD reduction was observed. In donor hearts, $\beta_{1^{-}}$ agonist induced a significant APD reduction in all layers. This demonstrates that the $\beta_{1}$-AR pathway is desensitized in failing hearts (Figure 3D).

We aimed to determine the molecular mechanism underlying the layer-dependency of $\beta_{2^{-}}$ stimulation by quantifying $\beta_{2}$-AR protein expression. In contrast with previous findings ${ }^{16}$, we observed that, in donor hearts, the expression of $\beta_{2}$-ARs was higher in the endocardium compared with the epicardium, with a $\beta_{2}$-AR-epi $/ \beta_{2}$-AR-endo ratio less than one. In failing hearts, the expression of $\beta_{2}$-ARs was reversed: higher expression is observed in the epicardium than the endocardium (Figure $3 \mathrm{E}$ ) and a $\beta_{2}$-AR-epi $/ \beta_{2}$-AR-endo ratio greater than one. The reversed transmural gradients in $\beta_{2}$-AR protein expression may explain the reversed endocardial-to-epicardial gradient effect of $\beta_{2}$-stimulation on APD in failing hearts.

Phosphorylation of $\beta_{2}$-AR by PKA was reduced in failing human hearts- $\beta$ stimulation controls electrophysiological targets primarily via the stimulatory G-proteins 
$\left(\mathrm{G}_{\mathrm{s}}\right)$ and downstream protein kinase A (PKA). Unlike $\beta_{1}$-AR, which couples only to $\mathrm{G}_{\mathrm{s}}, \beta_{2^{-}}$ AR can couple either to $G_{s}$ or to inhibitory G-protein $\left(G_{i}\right) \cdot{ }^{15}$ Phosphorylation of $\beta_{2}$-AR by PKA can switch its coupling from $\mathrm{G}_{\mathrm{s}}$ to $\mathrm{G}_{\mathrm{i}}{ }^{17}$ Therefore, we assessed $\beta_{2}$-AR phosphorylation at PKA-phosphorylation site Ser345/Ser346 ${ }^{18}$ and found $\beta_{2}$-AR phosphorylation was significantly lower in failing compared with donor hearts (Figure 3F), suggesting greater coupling to $G_{s}$ in failure.

Effect of $\beta_{2}$-AR stimulation on $\triangle \mathrm{CaD}-\mathrm{We}$ recorded CaT simultaneously with AP to generate $\mathrm{CaD}$ maps (Figure $4 \mathrm{~A}-\mathrm{B}$ ). $\mathrm{CaD}$ values at $80 \%$ of $\mathrm{Ca}$ relaxation are shown in Supplemental Figure 4. $\mathrm{CaD}$ shortening $(\triangle \mathrm{CaD})$ was quantified in order to evaluate the effects of $\beta$-stimulation on Ca reuptake. $\beta_{1}$-agonist treatment induced significantly greater $\mathrm{CaD}$ reductions in donor compared to failing hearts across the transmural wall (Figure 4D; the effect size between donor and failing hearts was transmurally homogeneous, as the group effect was strongly significant $(\mathrm{p}=0.0007)$ while the group-layer interaction was not significant $(\mathrm{p}=0.66)$. In contrast, $\beta_{2}$-agonist treatment shortened $\mathrm{CaD}$ to a similar degree in both donor and failing hearts (Figure $4 \mathrm{C}$ ). Interestingly, stimulation with the $\beta_{2}$-agonist did not induce similar transmural spatial differences as those observed with APD reduction.

Transmural gradient of CaD-APD-The CaD-APD is a measure of the period during which the intracellular calcium concentration $\left(\left[\mathrm{Ca}^{2+}\right]_{\mathrm{i}}\right)$ remains at a high level after the membrane has been completely repolarized (schematic in Figure 4E). Prolongation of this period, defined as $\Delta(\mathrm{CaD}-\mathrm{APD})=(\mathrm{CaD}-\mathrm{APD})_{\text {stimulation } \beta^{-}}(\mathrm{CaD}-\mathrm{APD})_{\text {baseline }}$, could lead to a window where the $\left[\mathrm{Ca}^{2+}\right]_{\mathrm{i}}$ is higher after $\beta$-stimulation than at baseline (green arrows in Figure 4E) during the period of complete membrane repolarization. In this vulnerable window, DADs may occur due to inward currents from the Na-Ca exchanger (NCX) while it extrudes $\mathrm{Ca}^{2+}$. In failing hearts, we observed prolongation of the $\Delta(\mathrm{CaD}-\mathrm{APD})$ after $\beta_{2}-$ stimulation in the epicardium $(-42.8 \pm 10.7 \mathrm{~ms}$ for donor versus $13.6 \pm 6.9 \mathrm{~ms}$ for failing, $\mathrm{p}$ $=0.003)$ and midmyocardium $(-32.0 \pm 10.1 \mathrm{~ms}$ for donor versus $7.1 \pm 7.2 \mathrm{~ms}$ for failing, $\mathrm{p}$ $=0.0074)$ but not in the endocardium $(-23.1 \pm 8.0 \mathrm{~ms}$ for donor versus $-31.6 \pm 12.1 \mathrm{~ms}$ for failing, $\mathrm{p}=0.53$ ), where negative values indicates that $\mathrm{CaD}-\mathrm{APD}$ value after stimulation is shorter than baseline condition. In donor hearts, prolongation of $\Delta(\mathrm{CaD}-\mathrm{APD})$ after $\beta_{2^{-}}$ stimulation was not observed (Figure 4F). Also, there were no significant differences in transmural $\Delta(\mathrm{CaD}-\mathrm{APD}) \mathrm{s}$ in either donor or failing hearts after $\beta_{1}$-stimulation (Figure $4 \mathrm{G}$ ).

$\beta_{2}$-AR stimulation altered CaT morphology-To evaluate changes in CaT morphology, we quantified the $\mathrm{CaD}_{30} / \mathrm{CaD}_{80}$ ratio. ${ }^{11} \mathrm{We}$ observed that the ratio is greater at baseline in failing hearts than in donor hearts throughout the transmural wall, as previous findings have also shown ${ }^{15}$ (Figure $\left.5 \mathrm{~A}\right)$. Treating with $\beta_{2}$-agonist equalized the ratio $(0.55 \pm$ 0.02 versus $0.55 \pm 0.01$, ns after $\beta_{2}$-stimulation; and $0.52 \pm 0.015$ versus $0.59 \pm 0.009, \mathrm{p}=$ 0.003 at baseline; donor vs. failing), which indicates a change in CaT morphology (Figure $5 \mathrm{~B}$ and $\mathrm{E}$ ). While the ratio in failing hearts was still greater than in donor hearts after treatment with the $\beta_{1}$-agonist, transmural heterogeneity was eliminated in both groups (Figure 5C and F); this is demonstrated by a strongly significant group-layer interaction at baseline ( $p<0.0001)$, but the absence of a significant group-layer interaction after treatment with $\beta_{1}$-agonist $(\mathrm{p}=0.076)$. 
We analyzed $\mathrm{Ca}^{2+}$ release and extrusion to determine the period in which $\beta_{2}$-stimulation causes changes in $\mathrm{CaT}$ morphology. We did not observe a difference in $\mathrm{Ca}^{2+}$ release when evaluating the rise time of $\mathrm{CaT}$ (Supplemental Figure 5). The $\mathrm{Ca}^{2+}$ extrusion was analyzed by determining two phases: the early phase (fast $\mathrm{Ca}^{2+}$ decline component), as determined by $\mathrm{T}_{50}$ (period of time from CaT peak to $50 \%$ reuptake), and the late phase (slow $\mathrm{Ca}^{2+}$ decline component), as determined by the $\mathrm{Ca}^{2+}$ decay constant. The $\mathrm{T}_{50}$ average for all layers decreased after $\beta_{2}$-stimulation in both donor and failing hearts (Figure 5G). However, the $\mathrm{Ca}^{2+}$ decay constant was similar before and after $\beta_{2}$-stimulation in failing hearts (Figure $5 \mathrm{H}$ ), which suggests that abrogation of $\beta_{2}$ regulation of $\mathrm{Ca}^{2+}$ handling occurred mainly during the slow $\mathrm{Ca}^{2+}$ reuptake phase.

$\beta$-AR stimulation is necessary for ectopic activity in human heart failure-We counted premature ventricular contractions (PVCs) during 8 minutes of pacing (4 minutes of 0.5 and 4 minutes of $0.67 \mathrm{~Hz}$ ) and automaticity after pacing. We did not observe spontaneous automaticity or PVCs under any condition in donor hearts. In contrast, failing hearts exhibited both ectopic PVCs and automaticity in response to $\beta$-stimulation. Multiple types of PVCs were observed after $\beta_{2}$-stimulation. Figure 6A shows automaticity and PVCs with different morphologies originating from different locations recorded after $\beta_{2^{-}}$ stimulation in failing hearts. PVC\#1 represents a type of irregularly occurring PVC with small amplitude and slow upstroke that propagated through a limited area, as circled, before being terminated. According to the AP signal amplitude map, these PVCs originated from the midmyocardium. PVC\#2 represents another type of PVCs with greater amplitude and fast upstroke that can propagate through the transmural surface. This type of PVC originated from the endocardium (Figure 6A).

Next, we analyzed the underlying mechanisms and involvement of calcium overload in these two types of PVCs (\#1 and \#2) We used AP-CaT delay-map to measure the time difference between AP activation and Ca release. Figure 6B shows a PVC\#1 where the Ca release occurred prior to AP upstroke, which suggests that it was triggered by Ca release. Thus, the mechanism responsible for PVC\#1 may be a Ca-driven process enhanced by $\beta_{2}$-stimulation. In all hearts, Ca-driven PVCs only originated from the subepicardium and midmyocardium (Figure 7B), which were the transmural regions with prolonged CaD-APD.

PVC\#2 shared the same origin with a series of regular beats of enhanced automaticity. This automaticity originated from the endocardium with a rate of 33 beats/min and was eliminated by rapid delivery of Lugol's solution to the subendocardial surface, suggesting that these events are due to the Purkinje fiber automaticity. ${ }^{19}$ To test this hypothesis, we determined the presence of the Purkinje fiber marker $\mathrm{Cx} 40$ by immunofluorescence. ${ }^{20}$ Figure 6D top-right shows that the tissue from where the regular beats originated has high expression of $\mathrm{Cx} 40$ at the intercalated disk, further implicating that Purkinje fiber automaticity underlies the observed events. 


\section{$\beta$-AR stimulation increased occurrence of spontaneous PVCs and rapid pacing induced VT in failing hearts}

To confirm the described mechanisms of automaticity and ectopy, we used real-time pseudo-ECG and monophasic AP recordings to measure the occurrences of PVCs and pacing induced VT (Figure 7A). The number of PVCs and VTs dramatically increased after $\beta$-AR stimulation in failing hearts, but neither could be induced in donor hearts (Figure 7CE). Both $\beta_{1}$ - and $\beta_{2}$-stimulation induced more automaticity and PVCs in the endocardium as compared to other regions, which mainly originated in the Purkinje fibers. Unlike $\beta_{1^{-}}$, which induced automaticity in the Purkinje fibers only, $\beta_{2}$-agonist also induced PVCs in epicardium and midmyocardium in addition to endocardium, among which more than $44 \%$ were Ca-mediated. No Ca-driven PVCs were observed during $\beta_{1}$-stimulation (Figure 7B).

\section{Discussion}

Our data show, for the first time, a differential role of $\beta_{1}$ - and $\beta_{2}$-AR pathways in arrhythmogenesis in human heart failure. We show that in failing hearts, $\beta_{1^{-}}$and $\beta_{2^{-}}$ stimulation provoke automaticity and PVCs through at least two mechanisms. Both $\beta$-ARs increase automaticity in Purkinje fibers, but only $\beta_{2}$-AR stimulation causes Ca-driven PVCs. $\beta_{2}$-stimulation further enhances arrhythmogenic substrates via $\mathrm{CV}$ regulation, spatially heterogeneous repolarization, and $\mathrm{CaD}$. Importantly, $\beta_{2}$-stimulation increased the transmural difference between $\mathrm{CaD}$ and $\mathrm{APD}$, which facilitated the formation of DAD (Summarized in Figure 8B).

\section{Arrhythmogenesis due to $\beta_{2}$ - and $\beta_{1}$-stimulation during end-stage heart failure}

Desensitization of $\beta_{1}$-AR and preserved effects of $\beta_{2}$-AR have been previously reported in gene/protein expression, ${ }^{16}$ and cardiac mechanics ${ }^{9}$ in human endocardial biopsies from patients with heart failure. We mapped the entire transmural LV and found sensitization of $\beta_{2}$-AR at the subendocardium and midmyocardium of failing hearts. Moreover, in contrast to attenuated $\beta_{1}$-regulation in failing hearts, $\beta_{2}$-regulation is not only sensitized but also remodeled at the molecular and tissue level. This remodeling develops an arrhythmic substrate involving conduction, repolarization, and $\mathrm{Ca}$ handling in end-stage heart failure. Although $\beta_{2}$-stimulation affected CV similarly in failing and donor hearts, the average anisotropy was increased only in failing hearts. Due to this increase, the impulse propagates slowly in the transverse direction but rapidly in the longitudinal direction, which may lead to conduction block ${ }^{21}$, while $\beta_{1}$-stimulation lowers such vulnerability by decreasing the anisotropy. In addition, $\beta_{2}$-stimulation (but not $\beta_{1}$-stimulation) induced remodeling of repolarization in time- and space-domains such as altered refractoriness and increased transmural heterogeneity, which would widen the vulnerability window and facilitate unidirectional block and reentrant arrhythmias. ${ }^{22}$ The additional arrhythmogenicity resulting from $\beta_{2}$-stimulation in the human failing myocardium provides mechanism for recent studies advocating the use of non-selective $\beta$-blockers or $\beta_{2}$-specific blockers for heart failure. ${ }^{7,8}$

\section{Switching of $\beta_{2}$-AR coupling from $G_{i}$ to $G_{s}$ in failing hearts}

$\beta_{2}$-AR stimulation resulted in significant reduction of APD across the transmural surface in failing compared to donor hearts, which suggests sensitization of $\beta_{2}$-AR in heart failure. 
This effect may result from a $\beta_{2}$-AR coupling switch from $G_{i}$ to $G_{s}$ proteins due to $\beta_{2}$-AR phosphorylation by PKA, which is also activated by $\mathrm{G}_{\mathrm{s} .}{ }^{17} \mathrm{We}$ observed significantly reduced $\beta_{2}$-AR phosphorylation in failing hearts, which suggests that $\beta_{2}$-ARs switch from $G_{i}$ to $G_{s}$ in heart failure. The switch from the protective $G_{i}$-dependent negative feedback control system to an unstable $\mathrm{G}_{\mathrm{s}}$-dependent positive feedback control system was rendered via remodeling of $\beta_{2}$-AR signaling (Figure $8 \mathrm{~A}$ ) in heart failure. Therefore, $\beta_{2}$-AR could enhance its control of EC coupling through switching from $G_{i}$ to $G_{s}$, as evident from enhanced $\triangle \mathrm{APD}$ in failing hearts. Our observations are in agreement with studies by the group of Harding. ${ }^{23}$ Their data showed a negative inotropic effect in failing human ventricular cardiomyocytes after application of $\beta_{2}$-AR antagonist ICI118,551. In contrast, cardiomyocytes from non-failing hearts showed no significant decreased inotropic effect in the presence of $\beta_{2}$-AR antagonist. These data further support our conclusion that, in heart failure, a greater proportion of $\beta_{2}$-AR couples with $G_{s}$. Furthermore, $\beta_{2}$-AR overexpression has a negative inotropic effect following treatment with ICI118,551 in mouse models. ${ }^{24}$ An increased proportion of $\beta_{2}-\mathrm{AR}-\mathrm{G}_{\mathrm{s}}$ coupling was reported in this transgenic mouse model, ${ }^{24}$ which, along with previously described work in human hearts ${ }^{23}$, lends support to our theory.

\section{$G_{i}$ to $G_{s}$ switch abrogates increase of NCX activity caused by $\beta_{2}$-stimulation}

In this study, we established that abrogation of $\beta_{2}$ regulation during the late phase of $\mathrm{Ca}$ extrusion accounted for $\mathrm{CaT}$ morphological change in heart failure. Between the major proteins (SERCA and NCX) that determine Ca extrusion, NCX has been recognized to contribute more during CaT late phase ${ }^{25}$ due to membrane potential being lower than the equilibrium potential of $\mathrm{NCX}\left(\mathrm{E}_{\mathrm{Na} / \mathrm{Ca}}\right) .{ }^{26}$ When $\mathrm{NCX}$ is over-expressed, $\beta_{2}$-stimulation upregulates its activity through $\beta_{2}$-AR coupling to $G_{i}$ protein. ${ }^{27}$ However, this increase is not supported by our result (Figure $5 \mathrm{G}$ ). Given that NCX is overexpressed in heart failure (Supplemental Figure 6), we postulate that reduced $\beta_{2}$-regulation may be due to decreased $\beta_{2}$-AR-G $\mathrm{G}_{\mathrm{i}}$ coupling.

\section{Transmural remodeling of $\beta_{2}$-AR regulation and DAD}

In contrast to APD regulation, $\beta_{2}$-AR system did not enhance $\mathrm{Ca}$ handling in failing hearts nor increased transmural $\mathrm{CaD}$ heterogeneity in either group. This disparity resulted in increased CaD-APD difference, which exacerbated DAD initiation. Here, we show DAD triggered PVCs associated with Ca release preceding AP upstroke in failing human LV myocardium. Interestingly, such calcium-mediated PVCs were only observed during $\beta_{2^{-}}$ stimulation. PVCs were localized at the epicardium and midmyocardium, where (CaD-APD) is prolonged. In heart failure, given NCX activity has not been further augmented by $\beta_{2}$ stimulation due to receptor uncoupling from $\mathrm{G}_{\mathrm{i}}$, the substrate (CaD-APD) could play a crucial role in DAD initiation (Figure 8A-B). In contrast, $\beta_{1}$-stimulation did not provoke DADs in failing hearts.

\section{Purkinje fibers are responsible for the majority of automaticity during $\beta$-stimulation in heart failure}

Low frequency PVCs in normal hearts are considered benign, but frequent PVCs in failing hearts are associated with LV dysfunction. ${ }^{28}$ In our study, the majority of PVCs originated from the $\mathrm{Cx} 40$ positive subendocardium. This suggests that PVCs evoked by $\beta_{1}$ - and $\beta_{2}$-AR 
stimulation arise in the Purkinje fibers in heart failure. Furthermore, it indicates that both subtypes may preserve the functional state of Purkinje fibers, different from that of working myocytes. Additionally, $\beta_{2}$-stimulation also evokes DAD-induced triggered activity in the subepicardium and midmyocardium. Therefore, only $\beta_{2}$-stimulation, and not $\beta_{1}$-stimulation, leads to increased arrhythmia vulnerability by facilitating arrhythmogenic triggers and substrates. This specificity suggests that non-selective or $\beta_{2}$-selective blockers may be more effective in treating and preventing arrhythmogenesis in heart failure than $\beta_{1}$-selective blockers.

\section{Limitations}

A possible limitation of our study is our use of specific $\beta_{1^{-}}$and $\beta_{2}$-agonists instead of a commonly used non-specific agonist combined with isoform-specific antagonists. We selected isoform-specific agonists Procaterol $(1 \mu \mathrm{mol} / \mathrm{L})^{12}$ and Xamoterol $(1 \mu \mathrm{mol} / \mathrm{L})^{13}$ because of their established specificity in the settings of heart failure. Furthermore, it was not possible to do a dose response investigation due to the limited number of human hearts and limited time available for experiments. We decided not to use an alternative approach based on Isoproterenol combined with $\beta_{1}$ - and $\beta_{2}$-blockers because we wanted to investigate the two types of receptors in the same heart, with access to a limited number of hearts. We acknowledge that lack of dose response data limits our ability to compare the relative contributions of $\beta_{1}$ and $\beta_{2}$ to EC coupling. Therefore, our comparisons were only between donor and failing hearts. Furthermore, we confirmed the selectivity of $\beta_{1}$ agonist Xamoterol, which had dramatic stimulation effects in donor but not in failing hearts, consistent with previous findings of desensitization of $\beta 1-\mathrm{AR}$ in heart failure. ${ }^{9}$

\section{Supplementary Material}

Refer to Web version on PubMed Central for supplementary material.

\section{Acknowledgments}

We thank Dr. Akshar Y. Patel for assistance in clinical data extraction and HOPE center at Washington University for the assistance in histology. We thank Dr. Michael Wallendorf for expert assistance in statistical analysis of the data. We also thank Dr. Bas Boukens and Maria Efimova for critical reading of the manuscript and helpful discussion.

Funding Sources: National Institutes of Health (NIH)/National Heart, Lung, and Blood Institute (NHLBI) grants R01HL114395 and R01HL085369, Translational Cardiovascular Biobank and Respository (TCBR) at Washington University (National Institutes of Health (NIH)/Clinical and Translational Science Award (CTSA) grant UL1TR000448), the Lucy \& Stanley Lopata Endowment, and the Richard J. Wilkinson Trust.

\section{References}

1. Wachter SB, Gilbert EM. Beta-adrenergic receptors, from their discovery and characterization through their manipulation to beneficial clinical application. Cardiology. 2012; 122:104-112. [PubMed: 22759389]

2. Singh B, Williams EV. Effects on atrial and ventricular intracellular potentials, and other pharmacological actions on cardiac muscle, of mj 1999 and ah 3474. Br J Pharmacol. 1970; 39:675687. [PubMed: 5485144]

3. Priori, SG.; Napolitano, C.; Cerrone, M. Basis and treatment of cardiac arrhythmias. Springer; Berlin Heidelberg: 2006. B-blockers as antiarrhythmic agents.; p. 235-266. 
4. Desantiago J, Ai X, Islam M, Acuna G, Ziolo MT, Bers DM, Pogwizd SM. Arrhythmogenic effects of beta2-adrenergic stimulation in the failing heart are attributable to enhanced sarcoplasmic reticulum ca load. Circ Res. 2008; 102:1389-1397. [PubMed: 18467626]

5. Litovsky SH, Antzelevitch C. Rate dependence of action potential duration and refractoriness in canine ventricular endocardium differs from that of epicardium: Role of the transient outward current. J Am Coll Cardiol. 1989; 14:1053-1066. [PubMed: 2551947]

6. Bristow MR, Hershberger RE, Port JD, Minobe W, Rasmussen R. Beta1- and beta2-adrenergic receptor-mediated adenylate cyclase stimulation in nonfailing and failing human ventricular myocardium. Mol Pharmacol. 1989; 35:295-303. [PubMed: 2564629]

7. Ruwald MH, Ruwald AC, Jons C, Alexis J, McNitt S, Zareba W, Moss AJ. Effect of metoprolol versus carvedilol on outcomes in madit-crt. J Am Coll Cardiol. 2013; 61:1518-1526. [PubMed: 23500269]

8. de Peuter OR, Lussana F, Peters RJ, Buller HR, Kamphuisen PW. A systematic review of selective and non-selective beta blockers for prevention of vascular events in patients with acute coronary syndrome or heart failure. Neth J Med. 2009; 67:284-294. [PubMed: 19841485]

9. Bristow MR, Ginsburg R, Umans V, Fowler M, Minobe W, Rasmussen R, Zera P, Menlove R, Shah $\mathrm{P}$, Jamieson S, Stinson EB. Beta 1- and beta 2-adrenergic-receptor subpopulations in nonfailing and failing human ventricular myocardium: Coupling of both receptor subtypes to muscle contraction and selective beta 1-receptor down-regulation in heart failure. Circ Res. 1986; 59:297-309. [PubMed: 2876788]

10. Lang D, Sulkin M, Lou Q, Efimov IR. Optical mapping of action potentials and calcium transients in the mouse heart. J Vis Exp. 2011; (55):3275. doi: 10.3791/3275. [PubMed: 21946907]

11. Lou Q, Fedorov VV, Glukhov AV, Moazami N, Fast VG, Efimov IR. Transmural heterogeneity and remodeling of ventricular excitation-contraction coupling in human heart failure. Circulation. 2011; 123:1881-1890. [PubMed: 21502574]

12. Bartel S, Krause EG, Wallukat G, Karczewski P. New insights into beta2-adrenoceptor signaling in the adult rat heart. Cardiovasc Res. 2003; 57:694-703. [PubMed: 12618231]

13. Engelhardt S, Grimmer Y, Fan GH, Lohse MJ. Constitutive activity of the human beta(1)adrenergic receptor in beta(1)-receptor transgenic mice. Mol Pharmacol. 2001; 60:712-717. [PubMed: 11562432]

14. Rohr S. Role of gap junctions in the propagation of the cardiac action potential. Cardiovasc Res. 2004; 62:309-322. [PubMed: 15094351]

15. Xiao RP, Avdonin P, Zhou YY, Cheng H, Akhter SA, Eschenhagen T, Lefkowitz RJ, Koch WJ, Lakatta EG. Coupling of beta2-adrenoceptor to gi proteins and its physiological relevance in murine cardiac myocytes. Circ Res. 1999; 84:43-52. [PubMed: 9915773]

16. Beau SL, Tolley TK, Saffitz JE. Heterogeneous transmural distribution of beta-adrenergic receptor subtypes in failing human hearts. Circulation. 1993; 88:2501-2509. [PubMed: 7902783]

17. Daaka Y, Luttrell LM, Lefkowitz RJ. Switching of the coupling of the beta2-adrenergic receptor to different g proteins by protein kinase A. Nature. 1997; 390:88-91. [PubMed: 9363896]

18. Nobles KN, Xiao K, Ahn S, Shukla AK, Lam CM, Rajagopal S, Strachan RT, Huang TY, Bressler EA, Hara MR, Shenoy SK, Gygi SP, Lefkowitz RJ. Distinct phosphorylation sites on the beta(2)adrenergic receptor establish a barcode that encodes differential functions of beta-arrestin. Sci Signal. 4:ra51. [PubMed: 21868357]

19. Damiano RJ Jr. Smith PK, Tripp HF Jr. Asano T, Small KW, Lowe JE, Ideker RE, Cox JL. The effect of chemical ablation of the endocardium on ventricular fibrillation threshold. Circulation. 1986; 74:645-652. [PubMed: 3742762]

20. Saffitz JE, Schuessler RB. Connexin-40, bundle-branch block, and propagation at the purkinjemyocyte junction. Circ Res. 2000; 87:835-836. [PubMed: 11073876]

21. Spach MS, Dolber PC, Heidlage JF. Influence of the passive anisotropic properties on directional differences in propagation following modification of the sodium conductance in human atrial muscle. A model of reentry based on anisotropic discontinuous propagation. Circ Res. 1988; 62:811-832. [PubMed: 2450697]

22. Kleber AG, Rudy Y. Basic mechanisms of cardiac impulse propagation and associated arrhythmias. Physiol Rev. 2004; 84:431-488. [PubMed: 15044680] 
23. Gong H, Sun H, Koch WJ, Rau T, Eschenhagen T, Ravens U, Heubach JF, Adamson DL, Harding SE. Specific beta(2)ar blocker ici 118,551 actively decreases contraction through a G(i)-coupled form of the beta(2)ar in myocytes from failing human heart. Circulation. 2002; 105:2497-2503. [PubMed: 12034656]

24. Milano CA, Allen LF, Rockman HA, Dolber PC, McMinn TR, Chien KR, Johnson TD, Bond RA, Lefkowitz RJ. Enhanced myocardial function in transgenic mice overexpressing the beta 2adrenergic receptor. Science. 1994; 264:582-586. [PubMed: 8160017]

25. Crespo LM, Grantham CJ, Cannell MB. Kinetics, stoichiometry and role of the na-ca exchange mechanism in isolated cardiac myocytes. Nature. 1990; 345:618-621. [PubMed: 2348872]

26. Bers, D. Excitation-contraction coupling and cardiac contractile force. Kluwer Academic Pub; 2001.

27. Sato M, Gong H, Terracciano CM, Ranu H, Harding SE. Loss of beta-adrenoceptor response in myocytes overexpressing the na+/ca(2+)-exchanger. J Mol Cell Cardiol. 2004; 36:43-48. [PubMed: 14734046]

28. Bogun F, Crawford T, Reich S, Koelling TM, Armstrong W, Good E, Jongnarangsin K, Marine JE, Chugh A, Pelosi F, Oral H, Morady F. Radiofrequency ablation of frequent, idiopathic premature ventricular complexes: Comparison with a control group without intervention. Heart Rhythm. 2007; 4:863-867. [PubMed: 17599667] 


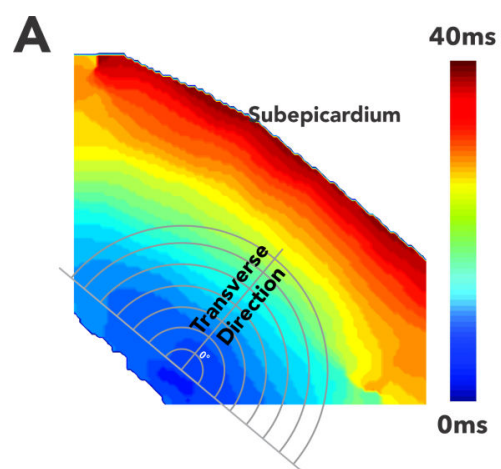

C

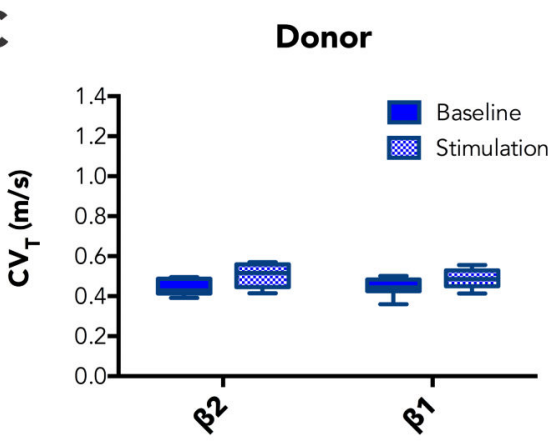

D

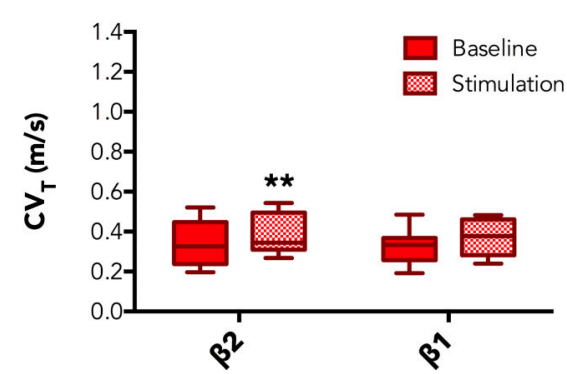

B

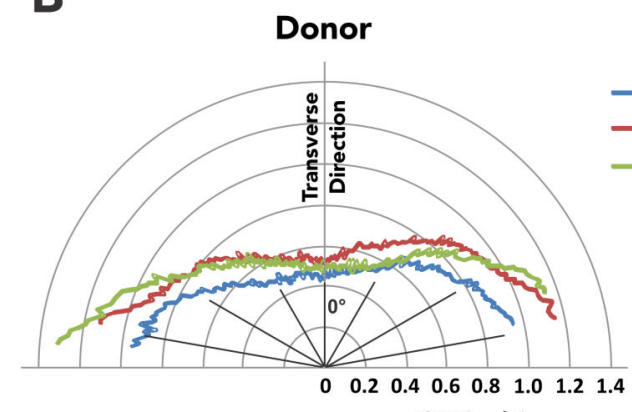

$\mathrm{CV}(\mathrm{m} / \mathrm{s})$

E

Donor

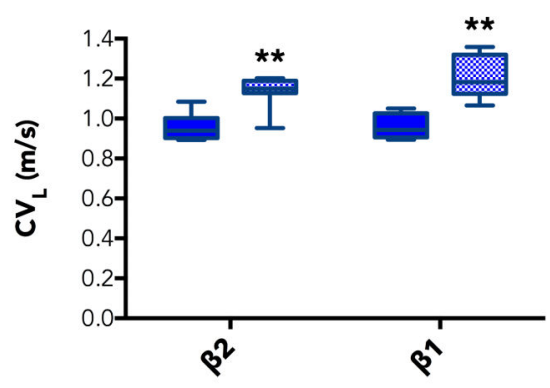

F

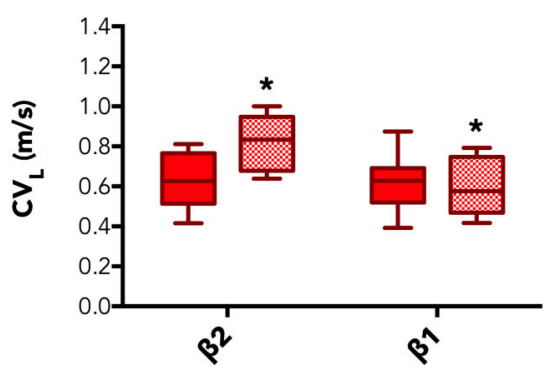

Failing

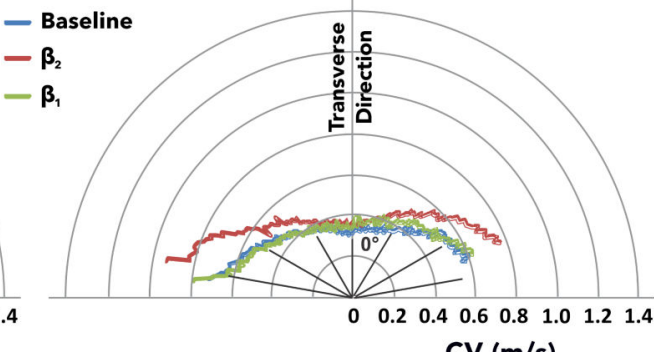

$\mathrm{CV}(\mathrm{m} / \mathrm{s})$

G

Donor $\quad \begin{array}{r}* p<0.05 \\ \star \star p<0.01\end{array}$

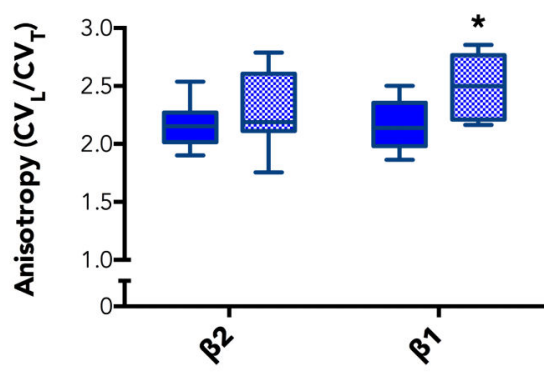

H

Failing

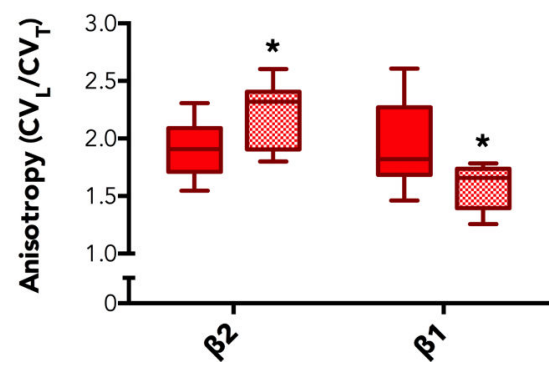

Figure 1.

Effects of $\beta$-stimulation on CV and anisotropy. A) Activation map illustrates CV measurements as a function of direction. B) Averaged wavefronts at each condition from donor and failing hearts are shown. $\mathrm{CV}_{\mathrm{L}}$ values for $\mathrm{C}$ ) donor and $\mathrm{D}$ ) failing hearts are shown compared with baseline values prior to stimulation. Graphs of $\mathrm{CV}_{\mathrm{T}}$ for $\mathrm{E}$ ) donor and $\mathrm{F}$ ) failing hearts with both stimulation conditions compared with baseline values. G) Donor and H) failing heart conduction anisotropy values. $\beta_{1}$-stimulation increased anisotropy in donor hearts but decreased it in failing hearts, while $\beta_{2}$-stimulation increased anisotropy in failing hearts. Box outlines show the $25^{\text {th }}$ and $75^{\text {th }}$ percentiles, and the middle lines indicate median values. Whiskers show the $5^{\text {th }}$ and $95^{\text {th }}$ percentiles. 
A

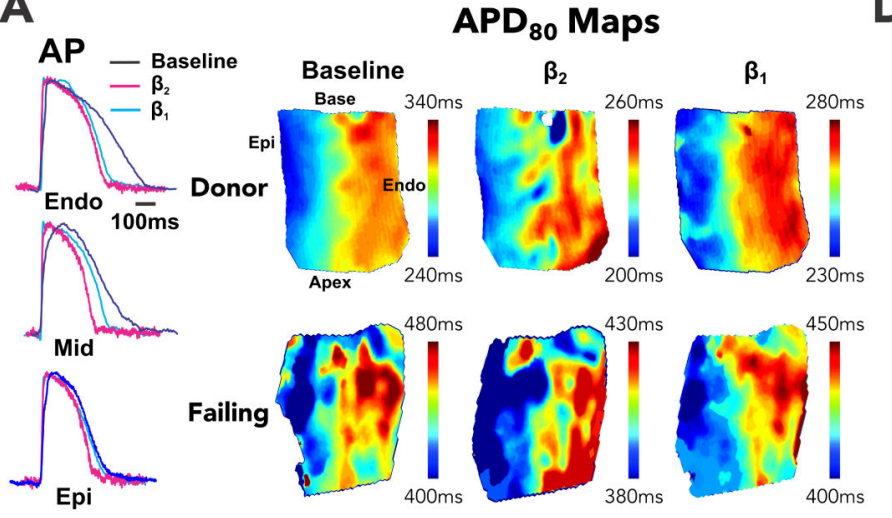

B

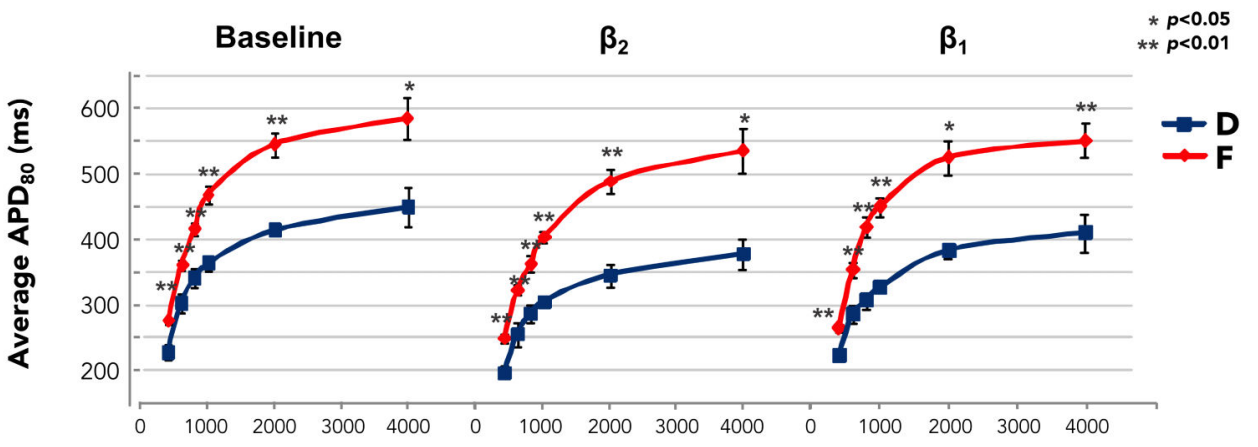

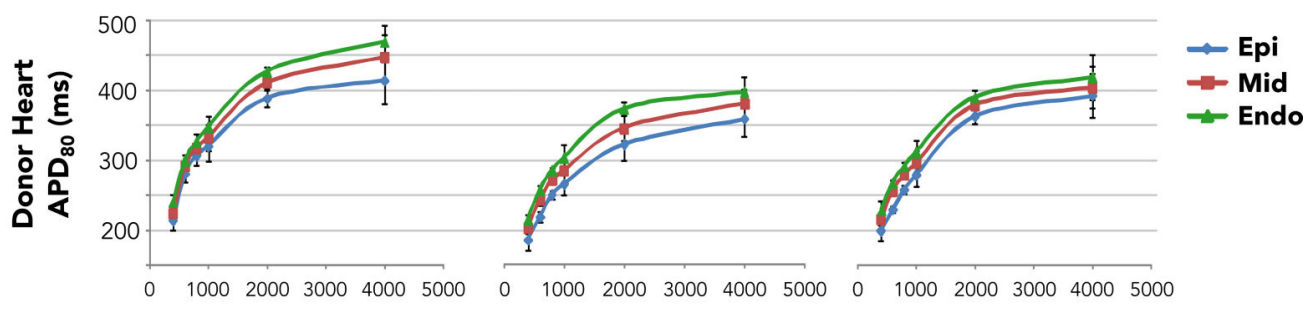

Pacing Cycle Length (ms)
Figure 2.

APD restitution curves and transmural gradients. A) Representative optical APs and APD maps recorded at $1 \mathrm{~Hz}$ pacing are shown for different conditions. B) Restitution curves of average APD over the entire field of view are shown. C) APD restitution curves from all layers are shown. D) Transmural gradients of repolarization $\left(\mathrm{APD}_{\mathrm{Endo}}-\mathrm{APD} \mathrm{Epi}_{\mathrm{i}}\right)$ at $1 \mathrm{~Hz}$ pacing are shown for $\beta_{2}$ (upper panel) and $\beta_{1}$-stimulation (lower panel). 
A

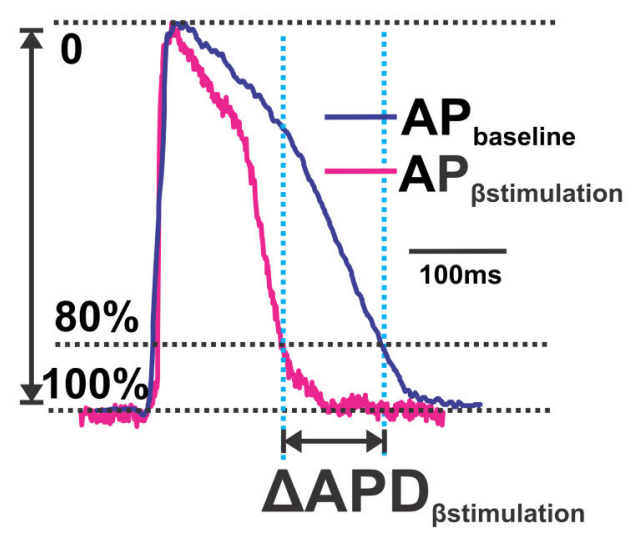

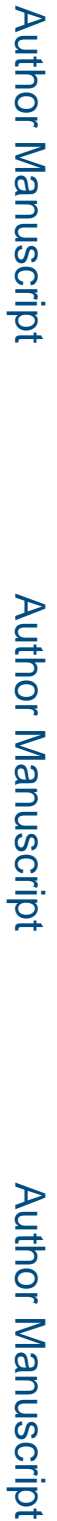
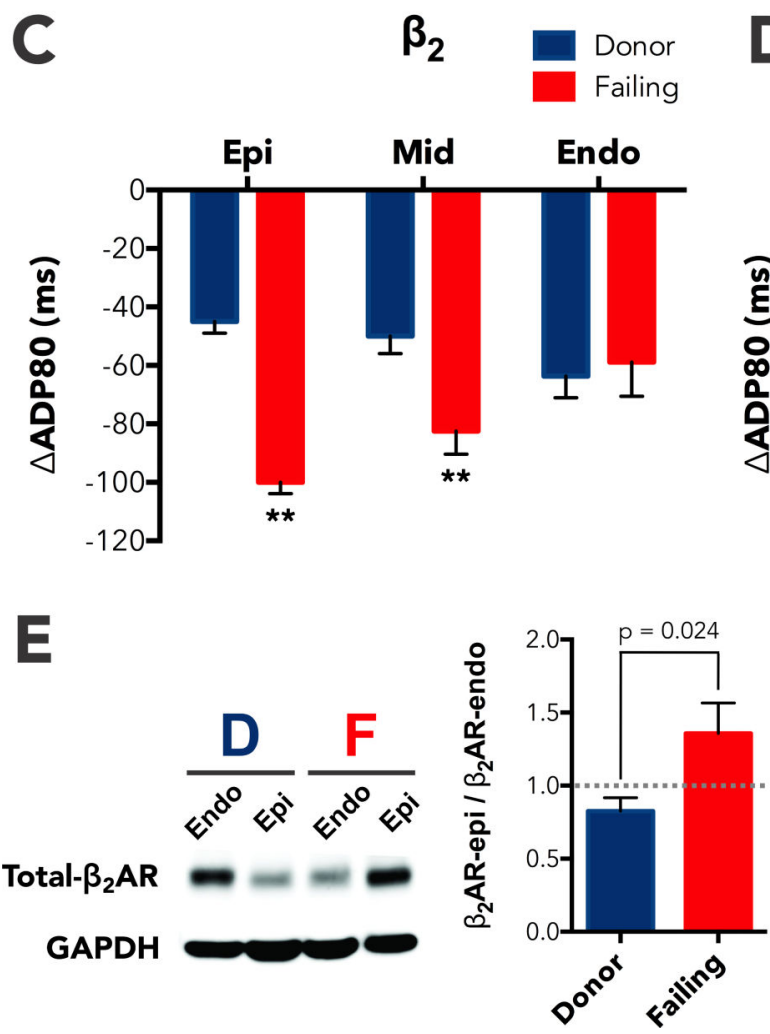

Figure 3.

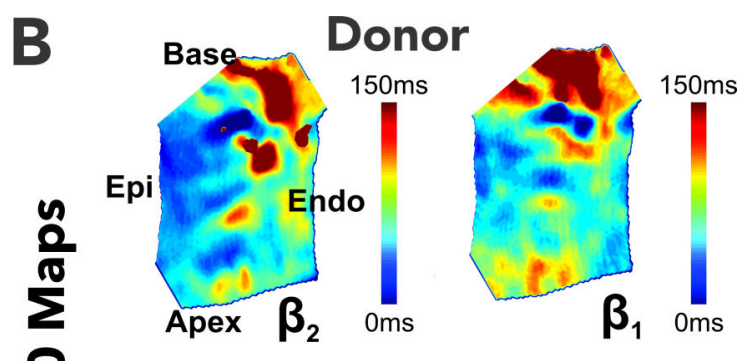

Failing

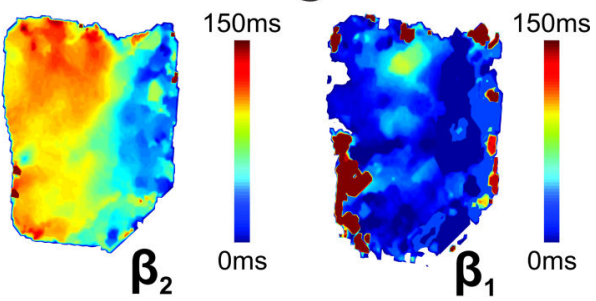

D

足
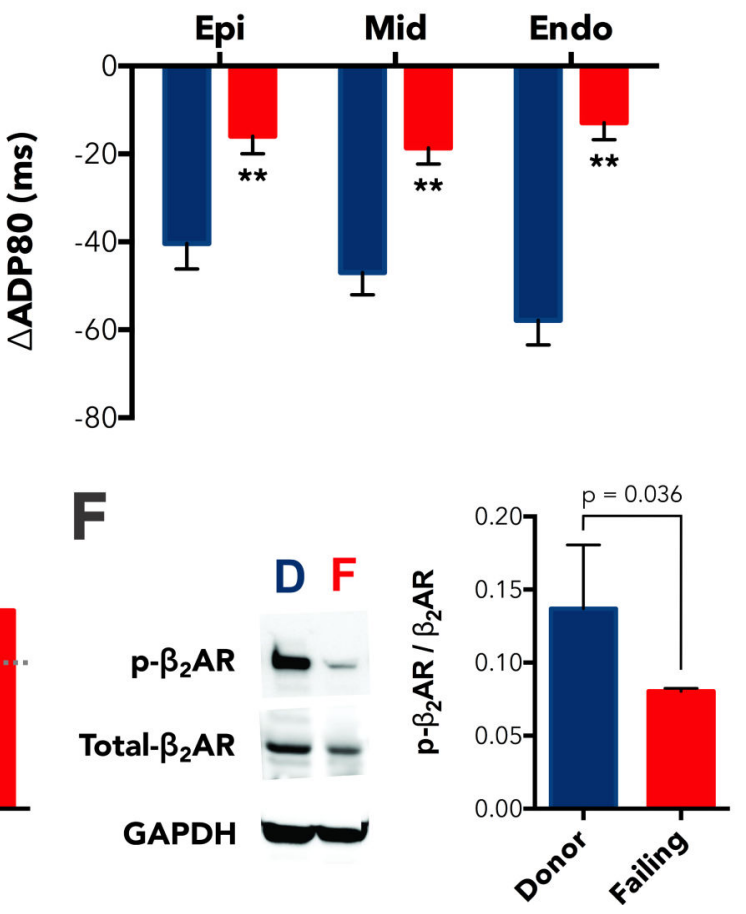

Effects of $\beta$-adrenergic stimulation on repolarization and remodeling of $\beta_{2}$-AR protein expression. A) AP signals showing that $\triangle \mathrm{APD}$ from $\beta$-stimulation are quantified as $\left.\mathrm{APD}_{\text {baseline }}-\mathrm{APD}_{\beta \text {-stimulation. }} \mathrm{B}\right)$ Representative $\triangle \mathrm{APD}$ maps of donor and failing hearts are shown. C) and D) Statistical analysis of $\triangle$ APD shows desensitization of $\beta_{1}$-AR and sensitization of $\beta_{2}$-AR at the endo and midmyocardium. Negative values indicate the reduction. E) Western blot shows a reversal of epi/endo gradient of $\beta_{2}$-AR protein expression in HF. F) Phosphorylation of $\beta_{2}$-AR is reduced in HF. 

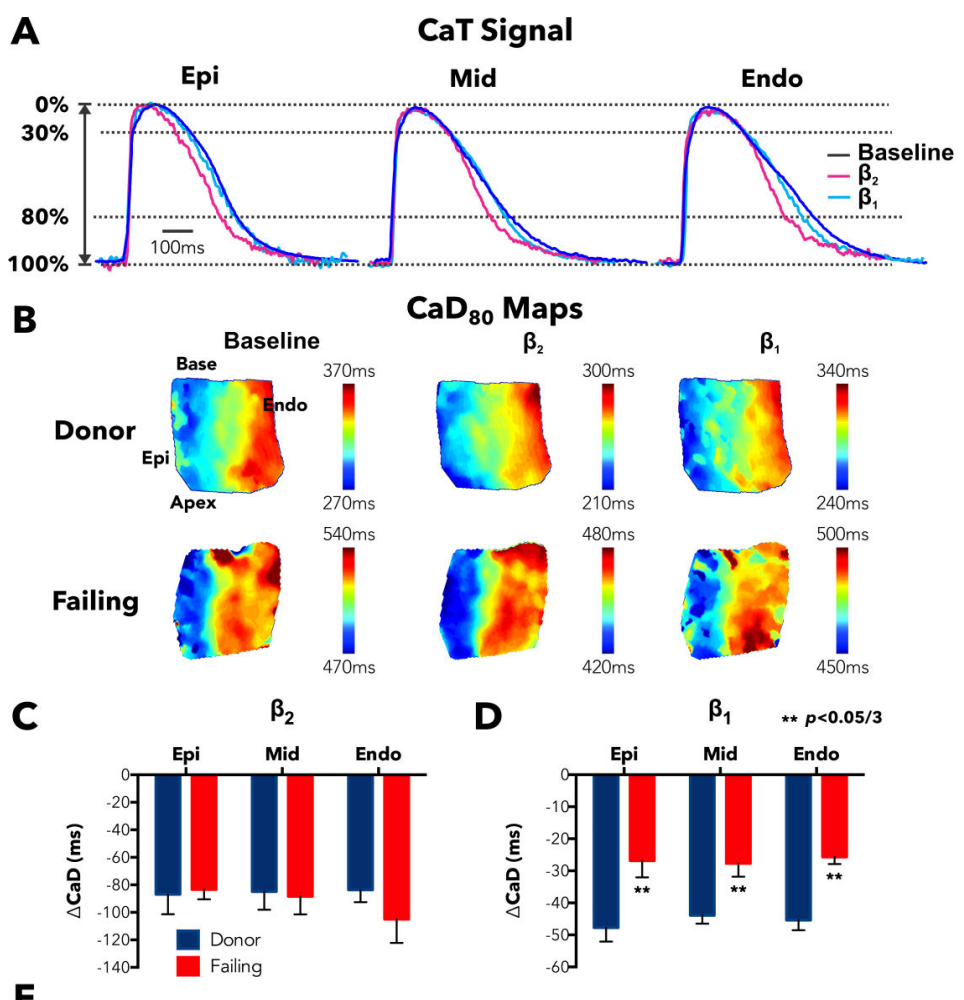

$\mathbf{E}$

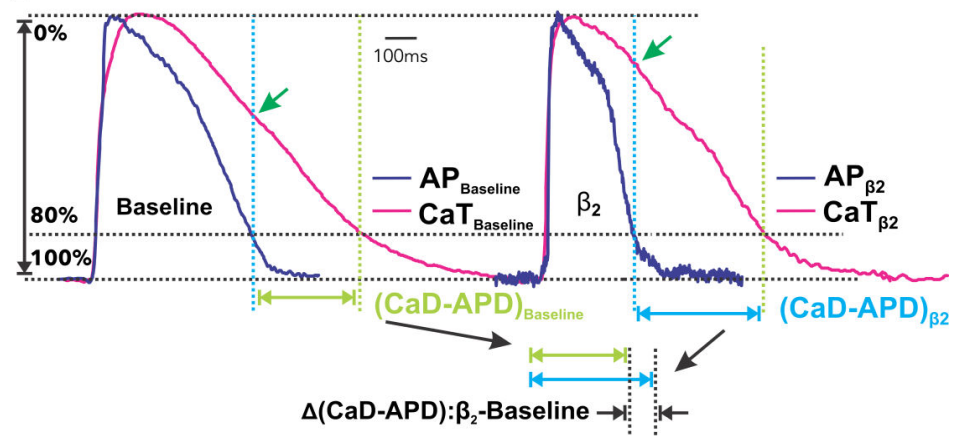

$\mathbf{F}$

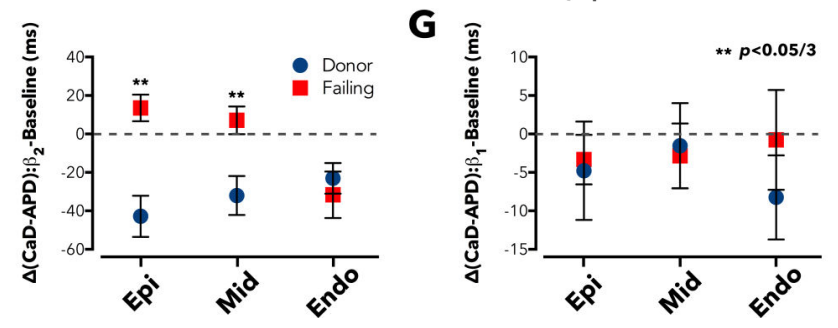

Figure 4.

Effects of $\beta$-stimulation on $\mathrm{CaT}$ and $\mathrm{CaD}$-APD. A) Representative CaT signals. B) Representative $\mathrm{CaD}_{80}$ maps. $\mathrm{C}$ ) and $\mathrm{D}$ ) Statistical analysis of $\Delta \mathrm{CaD}$ reveals partial desensitization of $\beta_{1}$-AR but not significant change of $\beta_{2}$-AR regulation in HF. E) Calculations of $\Delta(\mathrm{CaD}-\mathrm{APD})$ are shown. F) and G) Statistical analyses of $\Delta(\mathrm{CaD}-\mathrm{APD})$ are shown. 
D

\section{$\mathrm{CaD}_{30} / \mathrm{CaD}_{80}$ Ratio Maps}

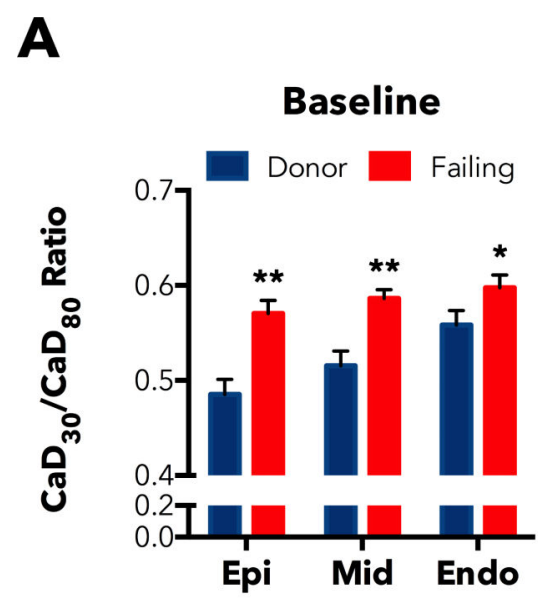

Baseline


E
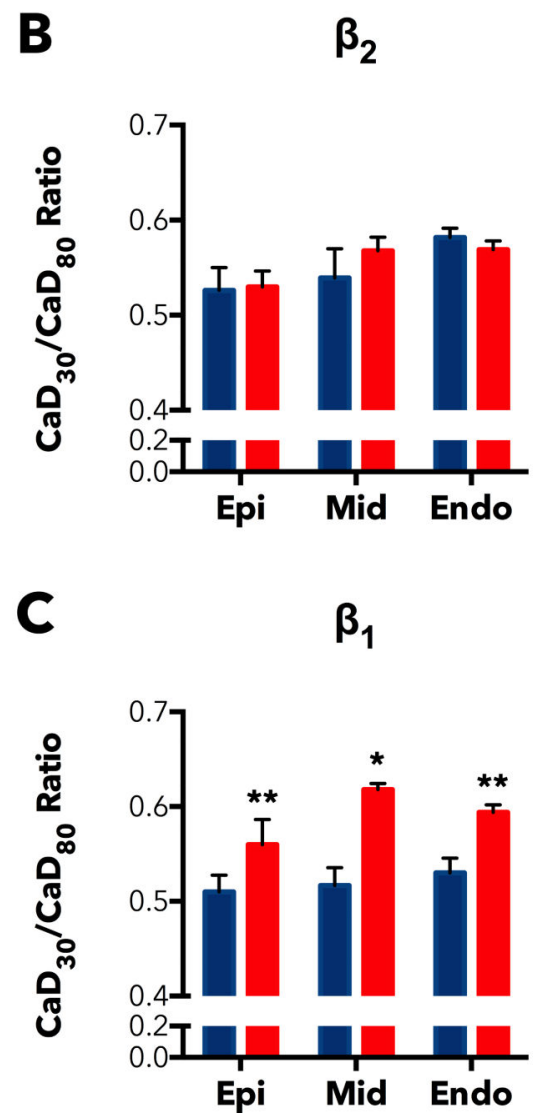

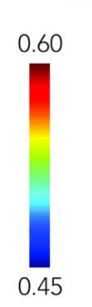

$\beta_{2}$
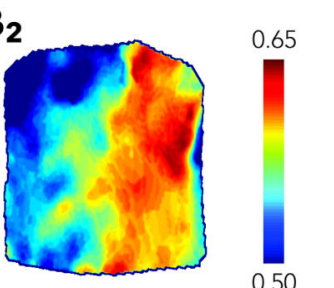

$\beta_{1}$
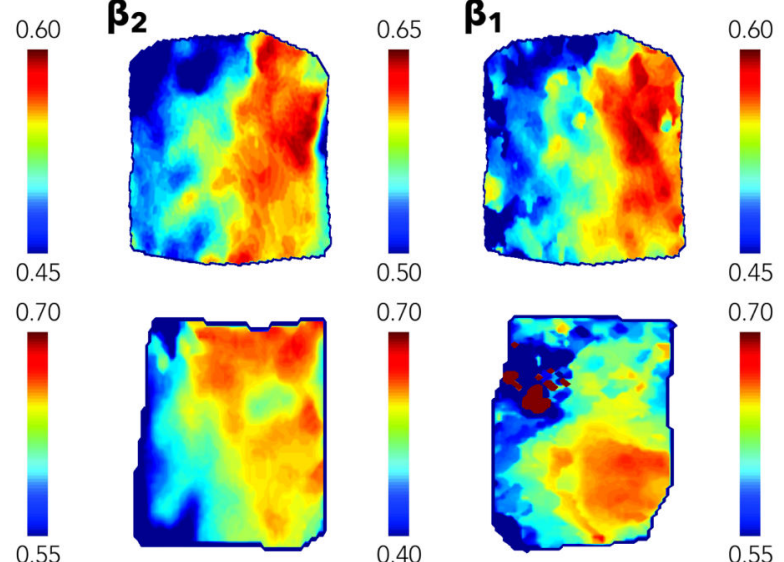

G
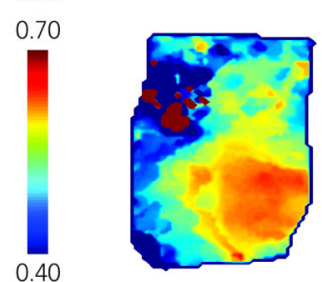

0.70

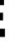

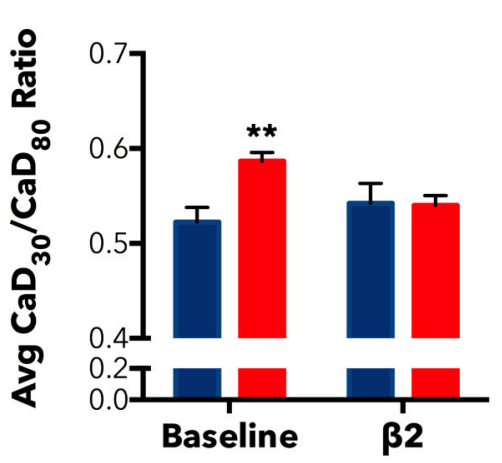

$\mathbf{F}$

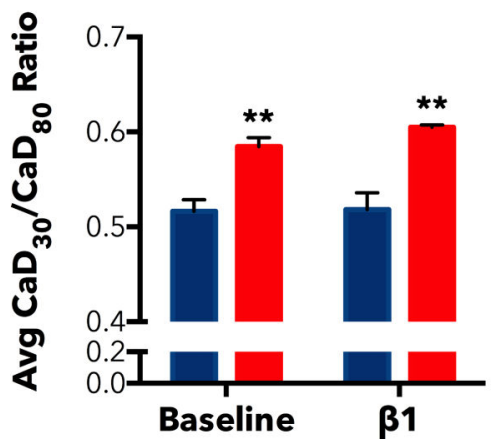

H

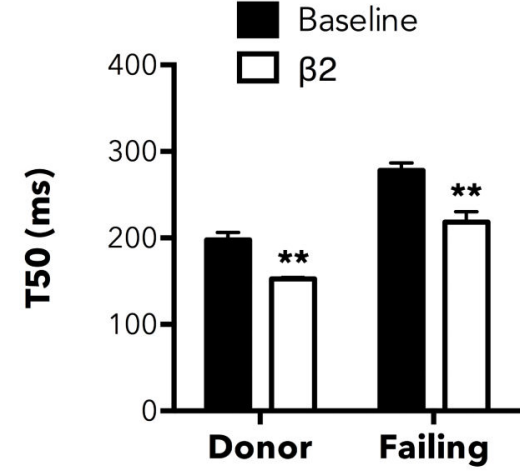

$* p<0.05$

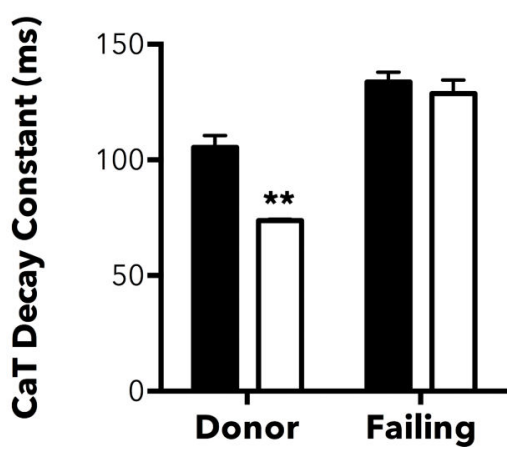

Figure 5.

Morphological remodeling of CaT. A) Baseline, B) $\beta_{2}$, and C) $\beta_{1} \mathrm{CaD}_{30} / \mathrm{CaD}_{80}$ ratios displayed for each transmural region, with $\mathrm{D}$ ) representative $\mathrm{CaD}_{30} / \mathrm{CaD}_{80}$ ratio maps. Transmurally averaged $\mathrm{CaD}_{30} / \mathrm{CaD}_{80}$ ratios for $\mathrm{E}$ ) $\beta_{2}$ and F) $\beta_{1}$ experiments. F) $\mathrm{T}_{50}$ and $\mathrm{G}$ ) $\mathrm{Ca}^{2+}$ decay constants at baseline and after $\beta_{2}$-stimulation are shown. 

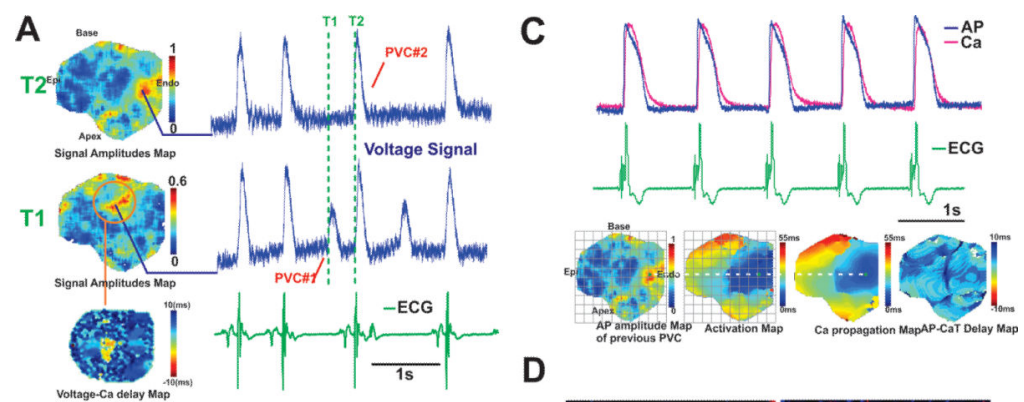

B
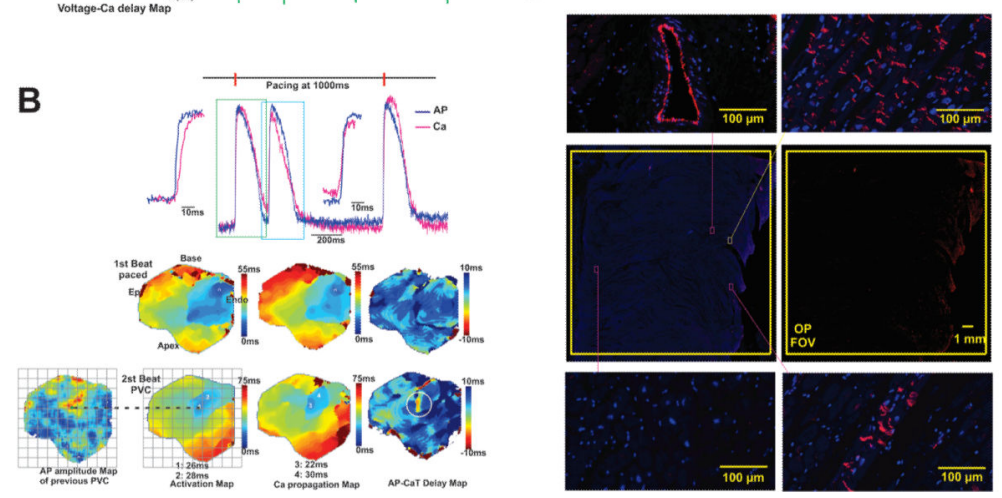

Figure 6.

Multiple mechanisms of automaticity and PVC in HF. A) Multiple types of PVCs were observed after $\beta_{2}$-stimulation. AP amplitude maps were constructed at T1 and T2 time points to determine the location of the origination of both types of PVCs B) Ca-driven PVC was confirmed by AP-CaT delay map and found to share the same location with PVC\#1. Activation times from two locations were labeled: PVC origination (Point \#1 and 3 on AP and CaT activation map, respectively) and an adjacent location (\#2 and 4). Activation time of 3 (CaT, 22ms) is earlier than 1 (AP, 26ms), and this difference is reversed shortly after AP propagated: activation time of 2 (AP, 28ms) is before 4 (CaT, 30ms). C) Enhanced automaticity shared the same location as PVC\#2. D) Immunofluorescence staining of Cx40 and DAPI. Cx40 staining was observed in coronary vascular endothelium (Top-left) and endocardium (Middle-right). The staining is negative in epicardium (Bottom-left). The region of enhanced automaticity (small yellow box) expressed Cx40 (Top-right). Intramural tract branch of Purkinje fibers penetrating into myocardium was also observed (Bottomright). 


\section{A \\ Rapid pacing induced VT}

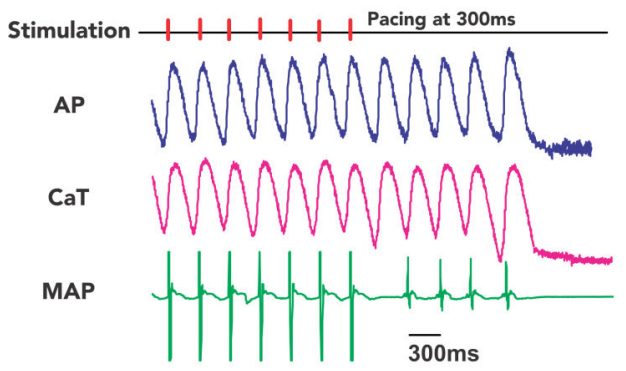

B

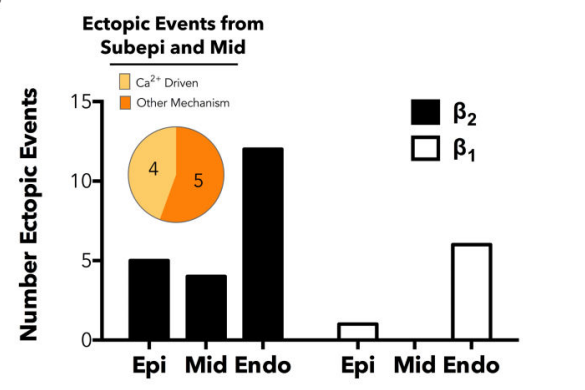

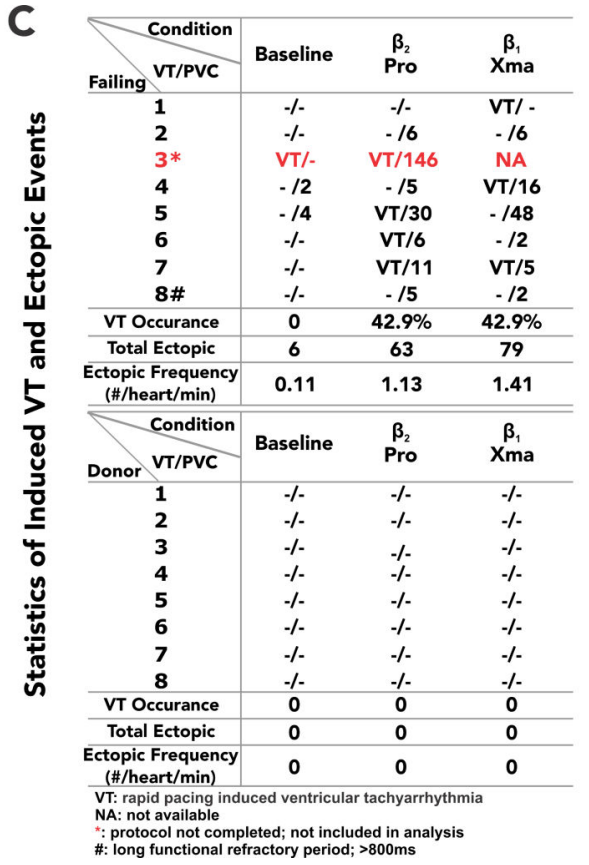

D

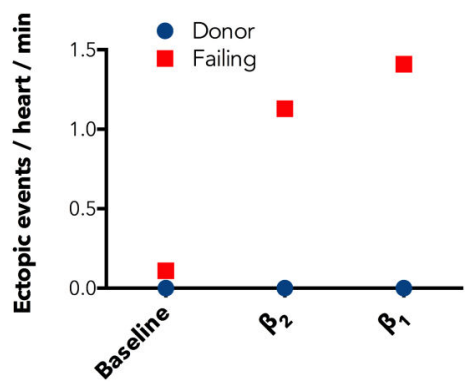

E

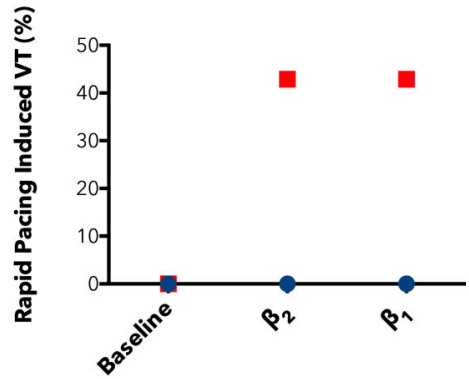

Figure 7.

Ectopic activity characterized by location and frequency. A) Representative rapid pacing induced ectopic activity (VT) with AP, CaT, and MAP are shown. B) Location distribution of ectopic activity. C) Statistics of induced VT and ectopy. D) and E) Expanded view of ectopy and VT occurrence are shown. 


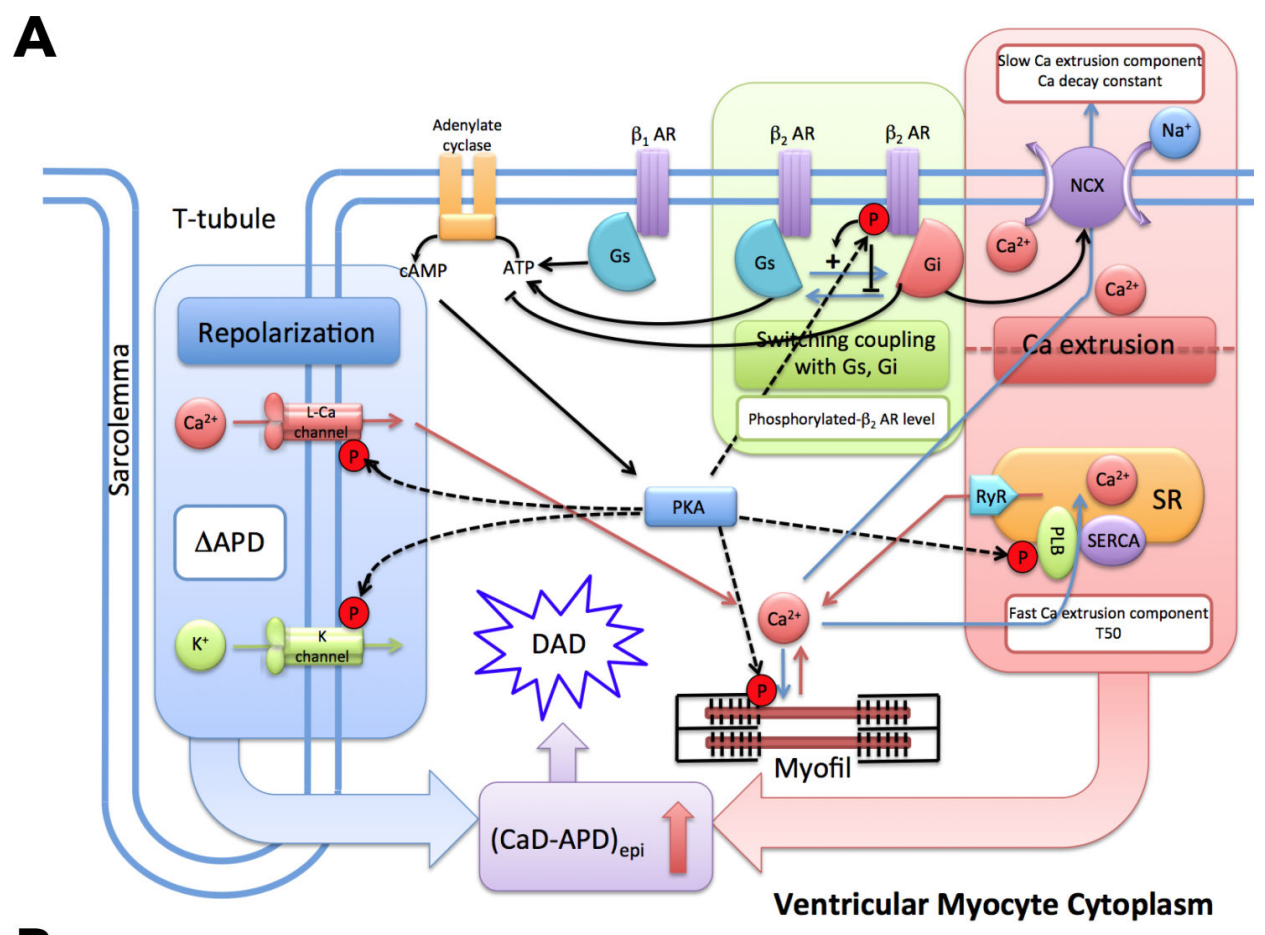

B

Failing LV Wedges
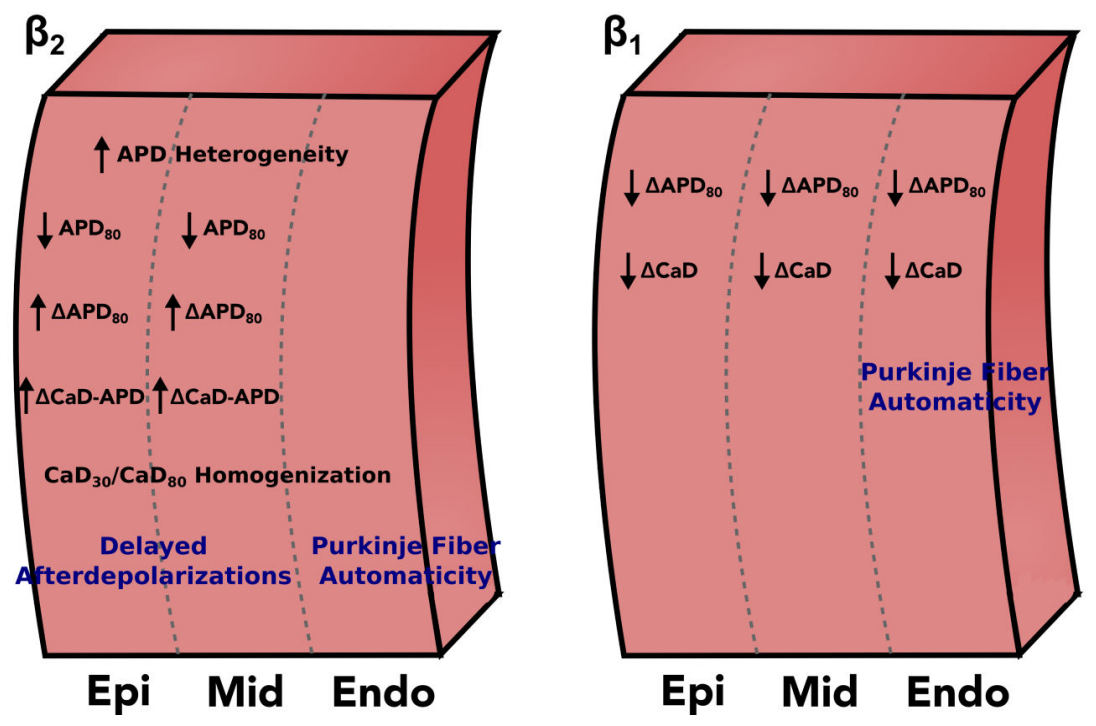

Figure 8.

Illustrated schematic summaries of $\beta$-AR signaling remodeling and electrophysiological effects in heart failure. A) Schematic highlighting the switch of $\beta_{2}$-AR coupling from $G_{i}$ to $\mathrm{G}_{\mathrm{s}}$ proteins, which alters downstream regulation from a negative to a positive feedback control system. B) Summary graphic of LV electrophysiologic responses to $\beta_{2^{-}}$and $\beta_{1^{-}}$ stimulation in $\mathrm{HF}$. 